\title{
The Viewpoints of Sexually Active Single Women About Premarital Sexual Relationships: A Qualitative Study in the Iranian Context
}

\author{
Azam Rahmani, ${ }^{1}$ Effat Merghati-Khoei, ${ }^{2}$ Lida Moghaddam-Banaem,, ${ }^{1, *}$ Ebrahim Hajizadeh, ${ }^{3}$ \\ and Ali Montazeri ${ }^{4}$ \\ ${ }^{1}$ Department of Midwifery and Reproductive Health, Faculty of Medical Sciences, Tarbiat Modares University, Tehran, IR Iran \\ ${ }_{2}^{2}$ Iranian National Center of Addiction Studies (INCAS), The Risk Behaviour Institution, Tehran University of Medical Sciences, Tehran, IR Iran \\ ${ }^{3}$ Department of Biostatistics, Faculty of Medical Sciences, Tarbiat Modares University, Tehran, IR Iran \\ ${ }_{4}^{4}$ Health Metrics Research Center, Iranian Institute for Health Sciences Research, Academic Centre for Education Culture and Research (ACECR), Tehran, IR Iran \\ ${ }^{*}$ Corresponding author: Lida Moghaddam-Banaem, Department of Midwifery and Reproductive Health, Faculty of Medical Sciences, Tarbiat Modares University, Tehran, IR Iran. \\ Tel:+98-2182883857, Fax: +98-2182884560, E-mail: moghaddamb@modares.ac.ir
}

Received 2014 August 31; Revised 2015 February 15; Accepted 2015 March 7.

\begin{abstract}
Background: Premarital sexual relationships could harm youth's health in terms of sexually transmitted infections or increased risk of unprotected sexual behaviors. Sexual abstinence has been recommended to prevent young adolescents from adverse outcomes of premarital sexual relationships.

Objectives: The aim of this study was to explore the viewpoints of sexually active single women about premarital sexual relationships in the Iranian context.

Patients and Methods: In this qualitative study, we recruited 41 young women aged 18 to 35 years. Data were collected using focus group discussions and individual interviews. We employed conventional content analysis to analyze the data. Multiple data collection methods, maximum variation sampling, and peer checks were applied to enhance the reliability of the findings.

Results: Eight themes emerged from the data analysis: 'acceptance of sexual contact in the context of opposite-sex relationships, 'sexual activity as a guarantee for keeping the boyfriend in the relationship', 'premarital sexual relationship as an undeniable personal right', 'having successful marriage in spite of premarital sexual relationships', 'virginity as an old fashioned phenomenon', 'love as a license for premarital sexual behaviors', 'goal-oriented relationship as a license for premarital sexual behaviors', and 'experiencing premarital sexual relationships in order to gain perfection'.

Conclusions: Results of this study could be applied to designing interventions, such as promotion of preventive beliefs or educational programs regarding premarital sexual relationships in conservative societies. These interventions could start within families and continue at schools and universities.
\end{abstract}

Keywords: Premarital Sex Behavior, Qualitative Research, Iran

\section{Background}

Risky sexual behaviors are commonly defined as behaviors that increase the risk of sexually transmitted infections and unintended pregnancies. They include having sex at an early age, multiple sexual partners, sex under the influence of alcohol or drugs, and unprotected sexual behaviors (1). Although premarital sexual relationships have not been defined as risky sexual behaviors in the international literature, they could be considered as risky behaviors in the Iranian context, as premarital intimate relationships are not religiously and socially acceptable in the Iranian culture (2). Cultural scenarios or social determinants of sexual behaviors recognize which behaviors are normal in any context and which ones are not (3). In fact, cultural factors could play an important role in defining risky sexual behaviors. Also, sexual health services and sex education are not provided for unmarried people in the Iranian context. Sexually active unmarried people, especially women, are vulnerable, since premarital sexual relationships could have inappropriate outcomes for women in terms of marriage (2).

Several studies have demonstrated that using condoms could reduce the likelihood of sexually transmitted diseases and related cancers, such as cancer of the cervix or penis, yet it could not totally omit the risk of these outcomes. Therefore, sexual abstinence before marriage is suggested as the best protective method for sexually transmitted diseases and their outcomes $(4,5)$. In addition there is evidence that adolescents who engage in premarital sexual activities may not experience the same quality marital relationship and stability as that of married people who abstained from premarital sexual activities (6). Thus, sexual abstinence has been recommended to prevent young adolescents from adverse outcomes of premarital sexual behaviors $(7,8)$.

Copyright (c) 2016, Zahedan University of Medical Sciences. This is an open-access article distributed under the terms of the Creative Commons Attribution-NonCommercial 4.0 International License (http://creativecommons.org/licenses/by-nc/4.0/) which permits copy and redistribute the material just in noncommercial usages, provided the original work is properly cited. 
Studies on premarital and risky sexual behaviors are crucial in the Iranian context for several reasons. According to the latest census conducted in Iran, young people make up about 20\% (15 million) of the population (9) and in Muslim societies, as is true within many religious traditions (10), they tend to postpone their marriage - the only institution that legally permits them to experience sexual activity and any relationship outside of marriage is culturally, religiously, and legally forbidden (2). However, despite the law, many young people may begin their sexual activities before marriage $(2,11)$. According to previous studies, 54 to $58 \%$ of male and female college students had been involved in premarital heterosexual relationships (12), and 8 to $12 \%$ of female college students had engaged in premarital sexual behaviors $(12,13)$. Also, a study on Iranian youth reported that $27.7 \%$ of these youth first experienced sexual intercourse at the age of fifteen; and $68.2 \%$ of sexually active teenagers had at least one risky sexual behavior (14). While previous reports have demonstrated the likelihood of rising risky sexual behaviors (15), there is no comprehensive sex education targeting youth in Iran. Also, risky sexual behaviors are the second main cause of human immunodeficiency virus (HIV) transmission in Iran (16).

\section{Objectives}

The aim of this study was to explore the viewpoints of sexually active single women about premarital sexual relationships in the Iranian context.

\section{Patients and Methods}

\subsection{Design}

In this qualitative study, we employed conventional content analysis since there was limited data in the field of premarital sexual relationships in the Iranian context and there was no preconceived hypotheses (17). A twostep qualitative approach, using focus group discussions (FGD) and individual interviews, was used in the study.

\subsection{Participants}

We recruited a group of young women $(n=41)$ aged 18 to 34 years old, who had volunteered to participate in this study. We initially planned to work on both males and females yet the ethics committee disapproved working on male participants because the primary researcher was female and cross-sex interviews on such a sensitive issue is not acceptable in the Iranian culture. Although we approached both sexually experienced and sexually inexperienced women, the reported results in the present study were mostly extracted from sexually experienced women.

\subsection{Data Collection}

We posted several announcements for a discussion group about 'premarital sexual relationships' that young women could attend. We employed focus group discussions as the primary means of data collection. The FGD was an appropriate technique to generate data from individual's values and to explore predominant social norms (18). In FGDs, we purposefully approached the participants regardless of their experiences of premarital sexual behaviors. The sessions were facilitated by defining premarital sexual behaviors, and using a non-structured inventory that began with an open-ended question: "how is the sexual life of single women in Iran?' Afterwards, based on the responses obtained from the participants, subsequent questions were built upon the discussion. Although Iran's constitution is based on Islamic laws and government policies are guided by Shari'a and Islamic principles, it is common for the new generation to not believe that speaking openly about sexuality is against religion or cultural norms (19). However, the participants with different levels of religiosity challenged each other's viewpoints in learning and experiencing sexuality. The focus group discussions enabled the investigators to identify the potential informants for individual interviews. Those women who had premarital sexual activity were identified during FGDs and invited for individual interviews. Although, women who had premarital sexual experience did not speak openly in the discussion groups, we identified these women when they spoke about their beliefs; for example, some of them believed that premarital sexual experience is a natural phenomenon and every woman, similar to a man, could enjoy this experience. Also, in order to have access to other young women with premarital sexual experiences, snowball sampling was used and additional young females were identified for individual interviews.

In the FGDs, participants challenged, questioned, and answered each other. Young women with different values, viewpoints, and experiences discussed the social aspects of premarital sexual relationships. In this step, data were generated according to predominant social norms. Young women with premarital sexual experiences could not speak about their experiences in FGDs, thus it was better to speak with these women during individual interviews.

In order to overcome the silence and privacy concerns in individual interviews, we: A) controlled our speaking style, look, gesture, and posture, B) consulted participants on their sexual life, if needed, C) ensured them that their private sexual experience will only be used for completing the research, D) gave a pseudonym to each woman to achieve anonymity, E) invited potential participants to dinner in order to establish rapport, and F) organized the questions from simple to complicated, so that participants did not feel we were intruding their private boundaries.

Sampling was continued with maximum variation to yield greater transferability and saturation of data (20). To achieve maximum variation in the data, informants were selected from different age groups, different socioeconomic status, high and low religiosity, and sexual experience levels. Also they had varying education levels. Most were living in dormitories and a few were liv- 
ing with their families at the time of interviews. In total, five focus group discussions were held and ten participants were interviewed individually. Data saturation was achieved after four focus group discussions and eight individual interviews; in fact, no new data were collected from the last two individual interviews and the last FGD.

\subsection{Data Analysis}

Inspired by Graneheim and Lundman's approach, we employed the qualitative content analysis. In this approach the most suitable unit of analysis is whole interviews or observational protocols (21). Data analysis commenced during the data collection. Each focus group discussion and individual interview was transcribed verbatim and analyzed before the next focus group discussion or interview. We achieved data comprehension thorough reading and re-reading. In the next step, the units of meaning were extracted from the statements. Data analysis was performed using line-by-line coding, and codes were created during repeated discussions between researchers. Themes emerged based on the codes with similar meanings.

\subsection{Rigor}

There was a second coder involved in the study, who was an expert in qualitative methods. We asked five of our participants to review the summary of the interviews and the findings (member check); these participants were chosen during the first FGDs and interviews. For confirmability of the findings, the substantive codes and themes were checked with four individuals, who were familiar with qualitative methods of content analysis (peer check) (20). Maximum variation sampling enhanced the transferability of data (22). Multiple data collection methods (focus group discussion and individual interview) were used to enhance dependability and credibility of the data (20).

\subsection{Ethical Considerations}

Approval to conduct the study was granted from the ethics committee of the school of medicine of the Tarbiat Modares University in 2011. All participants were given pseudonyms and were informed that participation in the study was voluntary, they could withdraw at any time, their confidentiality would be maintained, and none of the participants would be identified in any publications derived from the study. Informed written consent was obtained from the participants prior to focus group discussions and interviews.

\section{Results}

Eight themes emerged from the data analysis: 'acceptance of sexual contact in the context of opposite-sex relationships', 'sexual activity as a guarantee for keeping the boyfriend in the relationship', 'premarital sexual relationship as an undeniable personal right', 'having successful marriage in spite of premarital sexual rela- tionships', 'virginity as an old fashioned phenomenon', 'love as a license for premarital sexual behaviors', 'goaloriented relationship as a license for premarital sexual behaviors', and 'experiencing premarital sexual relationships in order to gain perfection'.

\subsection{Acceptance of Sexual Contact in the Context of Opposite-Sex Relationships}

Participants reported that they experienced a sense of guilt during their first sexual encounters. Also, most of them reported a sense of duality because they were doing something that opposed social norms, and in some cases opposed their own beliefs. After time progression, they felt more comfortable to engage in sexual relationships. In fact, they could accept the sexual contact as an inseparable part of their relationship, as some of the participants stated that they, themselves, welcomed sexual relationships. Sepideh, 21 years old, whose parents had talked to her about making a decision regarding premarital sexual relationships, reported that:

"My second boyfriend was frequently talking about sexuality issues; afterwards, I thought that experiencing premarital sexual behaviors may not be a special phenomenon and nothing will happen if I experience it. After that, I experienced sexual relationships and enjoyed it. Now, I think about why I should delete this enjoyable act from my life? If I love someone, I prefer to have sexual relationship with them and I have accepted sexual contact as part of my relationship."

\subsection{Premarital Sexual Relationship As an Undeniable Personal Right}

Most of the young women who had premarital sexual experiences believed that sexuality is a natural phenomenon and is a need that is required to be met. Thus, they experienced premarital sexual relationships more freely. This group of participants believed that it is ridiculous to abstain from sexual behaviors before marriage. They also stated that women could enjoy premarital sexual relationships as much as men and it is a mutual pleasure. Yasna, a 28 year-old woman, stated that:

"I believe that premarital sexual relationships are an undeniable right for everyone. Why do you eat an orange? You eat the orange because God has created it for you to eat. This is also true for premarital sexual relationships; God has created sexual relationships for you to experience and it is not important whether you experience it inside or outside of a marriage."

\subsection{Sexual Activity As a Guarantee for Keeping the Boyfriend in the Relationship}

Some of the young women believed that sexual relationships could keep their boyfriends in the relationship and encourage them to get married. In fact, these young women considered premarital sexual relationships as a trap 
for marriage. They believed that if emotional and sexual relationships are well established, their boyfriends will also love them and would want to experience sexual relationships only with them. Sahar, 25 years old, stated that:

"In my first opposite-sex relationship, I did not let my boyfriend engage in sexual relationships with me. In that relationship, my boyfriend left me. I thought if I had allowed us to engage in sexual relationships, I would be able to keep him in the relationship and convince him to get married. Now, I believe that I am not a bad girl and when God sends me an appropriate boy, he does not mind if I hug or kiss him. These behaviors could involve his emotions and feelings as well as mine."

\subsection{Having a Successful Marriage in Spite of Premarital Sexual Relationships}

Some participants believed that experiencing premarital sexual relationships is not an obstacle for them to have a successful marriage. Sima, 31 years old, stated that:

"There is a belief in the Iranian culture that men like to marry women who have not experienced premarital sexual behaviors. I think this belief is wrong. I have seen several women who had premarital sexual relationships and had a successful marriage afterward."

\subsection{Virginity As an Old Fashioned Phenomenon}

Some participants questioned the importance of virginity and stated that it is an old fashioned phenomenon because they believed virginity is not equal to female chastity. On one hand, a woman could experience different types of premarital sexual behaviors, other than vaginal, while she keeps her hymen intact. On the other hand, one's hymen could be damaged during an accident and it does not mean that she is not a virgin or a decent woman. In addition, some of the participants believed that they do not need to ask other people, such as family or community members, about keeping their virginity, because they believed the decision about keeping their virginity is a personal right. This viewpoint was observed not only among the participants but also among some families who allowed their daughters to experience any type of sexual behaviors. They had explained to their daughters that keeping virginity was not important for them. This viewpoint was evident by what the participants stated when describing people who had premarital sexual relationships as 'open-minded people'. Shima, 30 years old, stated that:

"After I realize that my boyfriend is a decent person, I decide to experience penetrative sexual relationships. I believe that what is more worthwhile than virginity is the individuals' personality, not a small part of the body [Hymen]."

\subsection{Experiencing Premarital Sexual Behaviors in Order to Gain Perfection}

Some participants believed that premarital sexual rela- tionships could be a way to reach perfection, relaxation and increased self-confidence. They reported that, occasionally, they preferred to experience sexual relationships in order to become relaxed after a mental fatigue or stressful event. In fact, the sexual relationship for these young women was a means to feel more comfortable and become relaxed. They believed that these occasional sexual relations could not satisfy them and they looked for a meaningful sexual relationship to gain perfection. Soraya, 28 years old, reported that:

"I like to have a successful sexual relationship, as I experience and feel perfection. In fact, when I communicate with my boyfriend and engage in sexual behaviors, I look for perfection, not merely sexual satisfaction. I mean I feel more confident and relaxed when I experience sexual relationships."

In fact some participants felt that a relation without sexual contact is not complete, and the sexuality part is what makes a relation worthwhile and whole.

\subsection{Love As a License for Premarital Sexual Behaviors}

Most of the participants who experienced premarital sexual relationships stated that they loved their boyfriends and they got involved in sexual behaviors because of their emotions. They stated that when you love someone, you like to touch his body and physical intimacy is a means of expressing your feelings. Although, premarital sexual relationship is not acceptable in the Iranian culture because of social and religious reasons, some participants believed that when two individuals fall in love and cannot live separately, there is no limitation for them to experience intimate relationships. Susan, 19 years old, stated that:

"Although experiencing premarital sexual relationships is taboo in the Iranian culture, if I love someone, I prefer to engage in sexual relationships, because it leads to a closer relationship"

\subsection{Goal-Oriented Relationship As a License for Premarital Sexual Behaviors}

Most participants reported that they usually engaged in an opposite-sex relationship if a number of criteria were met, e.g. if the relationship was goal-oriented. They defined goal-oriented relationships as long-term relationships or mutual intentions to get married. The purpose of the relationship, especially the intention to get married in the future, was a powerful motivation for them to engage in sexual behaviors because they thought sexual relationships could also help them know more about their boyfriend. They stated that they could evaluate if they are suitable cases for each other regarding marriage. Soheila, 25 years old, stated that:

"If I find someone qualified and eligible for marriage, I will engage in sexual behaviors because sexual relation- 
ships provide a situation for me to know my boyfriend better and make a better decision."

\section{Discussion}

The results of this study demonstrated that some beliefs could play an important role in motivating young women to engage in premarital sexual behaviors. Our study has provided a large amount of motivating and permissive beliefs regarding premarital sexual relationships.

In international studies, personal beliefs and attitudes are well documented as the strongest factors for premarital and risky sexual behaviors. Young people who have permissive attitudes toward premarital sexual activity are more likely to engage in premarital sexual behaviors more frequently and have more sexual partners. All of these beliefs, attitudes, and motivations can be considered as proximal factors because they are closely linked to risky and protective sexual behaviors and they influence those behaviors directly $(11,23-25)$. It should be noted that premarital relationships and sexual experiences, could also affect people's liberal attitudes (23). For example, having sex and using condoms may affect attitudes about having sex, perceptions of peer norms about sex, and perceived ability to use condoms. Thus, causality may operate in both directions (11).

Studies have demonstrated that if people have a virginity pledge, they are less likely to engage in premarital sexual relationships (11). Commitment to virginity is a predominant attitude and important in the Iranian culture, that is why our participants frequently discussed it. Something interesting in our study was that there was a certain sanctity attached to virginity; in fact, young women preferred to experience non-vaginal sexual behaviors, as if the hymen had a certain dignity for them. It seems that the importance of physical virginity for the Iranian society has caused an infusion of holiness to virginity and abstinence from vaginal sex, despite experiencing other types of sexual behaviors.

In the FGDs, participants frequently repeated that the importance of virginity has decreased, yet deep interviews with young women who experienced premarital sexual behaviors demonstrated that keeping virginity was still important. In fact, they did not believe that keeping virginity was worthwhile yet they tried to keep their hymen intact, despite experiencing non-vaginal sexual behaviors. Perhaps the type of importance has changed, as most young women who believed that keeping virginity is not a valuable thing were not willing to risk and lose their virginity. In fact, these young women had a sexual performance according to their sexual schema, which was formed by their family and society.

A qualitative study on Iranian young women showed that among the majority of young women who experienced premarital sexual behaviors, only a small fraction of these relationships had resulted in penetrative sex. In fact, they remained virgin to improve their opportunities for marriage. Although these young women might engage in more premarital heterosexual relations than other women, they may abstain from sex until marriage. These females might be better informed than their peers of the negative consequences of sex for their marriage prospects because of their better communication and closer relationships with their parents (2).

Results of this study could be applied for designing interventions, such as enhancing preventive beliefs or providing education regarding premarital sexual relationships in conservative societies. The current study had some limitations. Topics related to sexuality, such as premarital sexual relationship, are relatively personal and private, and are influenced by varying degrees of social, religious, moral, and legal norms and constraints. Thus, some people preferred not to speak about their sexual life, especially their sexual experiences. The main investigator was successful in decreasing this limitation by establishing rapport and trust. Also, because of religious and legal considerations, it is possible that only motivated or liberally minded young women participated, even though we invited all young women to participate in the study. Other researches on Iranian men with similar research questions are suggested to compare the findings.

\section{Acknowledgments}

This study was part of the first investigator's thesis for receiving a $\mathrm{PhD}$ degree in Reproductive Health, at Faculty of Medical Sciences, Tarbiat Modares University, Tehran, Iran. We would like to thank all young women who participated in this study.

\section{Footnotes}

Authors' Contribution:Azam Rahmani was the main investigator, designed the study, collected the data and wrote the first draft. Effat Merghati Khoei and Lida Moghaddam-Banaem were the study supervisors. Ebrahim Hajizadeh contributed to the statistics management and was the study consultant. Ali Montazeri was the study consultant.

Funding/Support:Tarbiat Modares University funded this PhD thesis.

\section{References}

1. Bachanas PJ, Morris MK, Lewis-Gess JK, Sarett-Cuasay EJ, Sirl K, Ries JK, et al. Predictors of risky sexual behavior in African American adolescent girls: implications for prevention interventions.J Pediatr Psychol. 2002;27(6):519-30. [PubMed: 12177252]

2. Farahani FK, Cleland J, Mehryar AH. Associations between family factors and premarital heterosexual relationships among female college students in Tehran. Int Perspect Sex Reprod Health. 2011;37(1):30-9. doi:10.1363/3703011. [PubMed: 21478086]

3. Gagnon JH, Simon W. Sexual conduct: The social sources of human sexuality. New Jersey: Transaction Publishers; 2011.

4. Balon R, Segraves RT. Clinical manual of sexual disorders. Washing- 
ton DC: American Psychiatric Pub; 2009.

5. Bomar PJ. Promoting health in families: applying family research and theory to nursing practice. US: Elsevier Health Sciences; 2004.

6. Ogunsola MO. Abstinence from premarital sex: a precursor to quality relationship and marital stability in subsequent marriage in Nigerian Society. Int J Psychol Stud. 2012;4(2):p228.

7. Kohler PK, Manhart LE, Lafferty WE. Abstinence-only and comprehensive sex education and the initiation of sexual activity and teen pregnancy. J Adolesc Health. 2008;42(4):344-51. doi: 10.1016/j. jadohealth.2007.08.026. [PubMed:18346659]

8. Kirby D, Lepore G, Ryan J. Sexual risk and protective factors, Factors affecting teen sexual behavior, pregnancy, childbearing and sexually transmitted disease: Which are important? ETR associates. 2005.

9. Iran-SCo. The overall results of General Population and Housing Census. 2011

10. Kotb H. Sexuality in Islam (PhD dissertation). Egypt: Maimonedes University; 2004.

11. Kirby DB, Laris BA, Rolleri LA. Sex and HIV education programs: their impact on sexual behaviors of young people throughout the world. J Adolesc Health. 2007;40(3):206-17. doi:10.1016/j.jadohealth.2006.11.143. [PubMed: 17321420]

12. Saboor-Parsa M, Tabatabaee A, editors. Religion, family and sexual behaviour in a university population.; 2nd Congress on Family and Sexual Disorders of Shahed University.; 2005; pp. 23-4.

13. Simbar M, Ramezani Tehrani F, Hashemi M. The reproductive health needs of the university students of Qazvin (in Persian). J Qazvin Univ Medic Sci Health Servi. 2003;28:5-13.

14. Mohammadi MR, Mohammad K, Farahani FKA, Alikhani S, Zare M, Tehrani FR, et al. Reproductive Knowledge, Attitude and Practice of Tehranian adolescent boys aged 15-18 years, 2002.J Reproduct Infertil. 2003;4(3)

15. Mohammad K, Farahani FK, Mohammadi MR, Alikhani S, Zare $\mathrm{M}$, Tehrani FR, et al. Sexual risk-taking behaviors among boys aged 15-18 years in Tehran. J Adolesc Health. 2007;41(4):407-14. doi: 10.1016/j.jadohealth.2007.05.003. [PubMed:17875467]

16. Nasirian M, Doroudi F, Gooya MM, Sedaghat A, Haghdoost AA Modeling of human immunodeficiency virus modes of transmission in Iran.J Res Health Sci. 2012;12(2):81-7. [PubMed: 23241516]

17. Hsieh HF, Shannon SE. Three approaches to qualitative content analysis. Qual Health Res. 2005;15(9):1277-88. doi: 10.1177/1049732305276687. [PubMed:16204405]

18. Krueger R, Casey M. Focuse Groups. 3rd ed. Thousand Oaks, CA: SAGE; 2000 .

19. Khoei EM. Language of Love in Culture of Silence: Iranian Women's Sexual Understandings and Sociocultural Context. New South Wales University, Sydney, Australia. 2006.

20. Speziale HS, Streubert HJ, Carpenter DR. Qualitative research in nursing: Advancing the humanistic imperative. Philadelphia: Lippincott Williams \& Wilkins; 2011

21. Graneheim UH, Lundman B. Qualitative content analysis in nursing research: concepts, procedures and measures to achieve trustworthiness. Nurse Educ Today. 2004;24(2):105-12. doi: 10.1016/j.nedt.2003.10.001. [PubMed: 14769454]

22. Holloway I, Wheeler S. Qualitative research in nursing and healthcare. Hoboken, New Jersey, United States: John Wiley \& Sons; 2013.

23. Farahani FK, Cleland J, Mehryar AH. Correlates and Determinants of Reproductive Behavior among Female University Students in Tehran.J Reprod Infertil. 2012;13(1):39-51. [PubMed: 23926523]

24. Alexander M, Garda L, Kanade S, Jejeebhoy S, Ganatra B. Correlates of premarital relationships among unmarried youth in Pune district, Maharashtra, India. Int Fam Plan Perspect. 2007;33(4):150-9. doi: 10.1363/ifpp.33.150.07. [PubMed: 18178539]

25. Abraham L, Kumar KA. Sexual Experiences and Their Correlates Among College Students in Mumbai City, India. Int Fam Plann Perspect.1999;25(3):139-52. doi:10.2307/2991963. 